\title{
EDUCATIONAL PROGRAM ASSOCIATED WITH THERAPEUTIC INTERVENTIONS SIGNIFICANTLY REDUCE PAIN AND FUNCTIONAL DISABILITY IN PATIENTS WITH CHRONIC SPINE PAIN
}

Fabiana Santos Tigre ${ }^{1}$, Maria Carolina Davel Lemos ${ }^{1}$, Luiz Paulo do Carmo Guanabens ${ }^{1}$, Patrícia Vieira Quadra Ribeiro ${ }^{1}$, Carla Vasconcelos Cáspar Andrade ${ }^{1}$, Danilo Rodrigues Ramos ${ }^{1}$, Gustavo Pinto de Oliveira Gomes ${ }^{1}$, Ana Catharina Nunes de Araújo ${ }^{1}$, Érica Vieira Serrano ${ }^{1}$, Valéria Valim¹, Maria Bernadete Renoldi de Oliveira Gavi1,*

1.Universidade Federal do Espírito Santo, Vitória (ES), Brazil.

*Corresponding author: mbernadetegavi@gmail.com

\section{BACKGROUND}

With the purpose of improving the treatment of chronic back pain and instituting adequate habits to prevent pain exacerbations, the Spine School Project was created, formed by an interdisciplinary team composed of rheumatologists and algologist, physiotherapists, physical education professionals and students from graduate and postgraduate courses at the Federal University of Espírito Santo (UFES).

\section{METHODS}

The aim was to evaluate whether a Pain Education program followed by a physical exercise protocol is more efficient in improving pain, functional capacity and flexibility than the Education group alone for patients with chronic spinal pain. The target audience consists of referrals from the Services of Rheumatology, Orthopedics and Pain Outpatient Clinic of the University Hospital of UFES. Lectures were given on causes of pain, neuroscience in physiotherapy, joint protection measures and energy conservation, as well as information on different types of treatment. Theoretical lectures took place 1 day a week, for 2 hours, for 4 consecutive weeks. Afterwards, the patients were included in the assistance protocol of specific physical exercises. Evaluations were carried out in 3 moments: before (AV1) and after the cycle of 4 lectures (AV2) and at the end of the exercise program (AV3). The Rolland Morris questionnaire was applied, flexibility was assessed by the Wells Bank, and pain intensity was assessed using the visual analog scale (VAS). Two homogeneous groups of patients were compared as follows: Group 1 (assessed education only) with 79 people, was studied in AV1 and AV2, Group 2 (school + exercises) with 62 people, was studied in AV1 and AV3. The difference between the means of the groups was analyzed using the Student's t-test for paired samples, considering the value of $p<0.05$ as statistically significant.

\section{RESULTS}

In group 1 there was a statistically significant difference $(p<0.0001)$ only for the Rolland Morris questionnaire between AV1 $(16.06 \pm 4.35)$ and AV2 (12.43 \pm 5.06$)$. In group 2 there was a statistically significant difference $(p<0.0001)$ for pain assessment between AV1 (VAS $=6.65 \pm 2.37$ ) and AV3 (VAS $=2.72 \pm 2.54)$, for the Rolland Morris questionnaire between AV1 $(16.06 \pm 4.35)$ and AV3 $(7.73 \pm 5.14)$ and also for the assessment of flexibility through the Wells Bank ( $p=0.03)$, AV1 (15.39 \pm 9.36$)$ and AV3 $(19.40 \pm 12.73)$.

\section{CONCLUSION}

Data analysis showed greater benefit in the group that participated in an interdisciplinary educational program associated with a physical exercise protocol when compared to the group that participated only in an Educational Program.

\section{KEYWORDS}

Spine school, Chronic spinal pain, Rehabilitation. 